This is the final peer-reviewed accepted manuscript of:

Barbera, E., Brini, F. A rational extended thermodynamics model for metal electrons in bounded domains. Ricerche mat 68, 37-56 (2019).

The final published version is available online at: https://doi.org/10.1007/s11587018-0377-9

Rights / License:

The terms and conditions for the reuse of this version of the manuscript are specified in the publishing policy. For all terms of use and more information see the publisher's website.

This item was downloaded from IRIS Università di Bologna (https://cris.unibo.it/)

When citing, please refer to the published version. 


\title{
A Rational Extended Thermodynamics Model for Metal Electrons in Bounded Domains
}

\author{
Elvira Barbera • Francesca Brini
}

Received: date / Accepted: date

\begin{abstract}
In this paper we introduce an 8-moment model for metal electrons, deduced through a Rational Extended Thermodynamics macroscopic approach. Afterwards, we focus on an electron gas confined in a bounded domain and discuss the assignment of the boundary data, taking into account also the wall effects.
\end{abstract}

Keywords Rational Extended Thermodynamics · Metal Electrons ·

Boundary conditions

\section{Introduction}

We present a model for the free electrons bounded within a metal. Following the original idea by Sommerfeld [1-4], we assume that the electrons in the metallic body are described as free fermion particles of mass $m$. Electrons can collide occasionally with a lattice ion (mass $M$ ), but their energy is unchanged by a collision, due to the large ratio $M / m$. In fact, the ions are modeled as rigid spheres, at rest at their lattice point and their density is uniform and constant. Moreover, we suppose that the electrons do not collide with each other.

In order to construct the model, we will refer to Rational Extended Thermodynamics (RET) [4,5], a macroscopic theory based on a different strategy

Dedicated to Professor Tommaso Ruggeri on his seventieth birthday.

E. Barbera

Department of Mathematical, Computer, Physical and Earth Sciences,

University of Messina, V.le F. D'Alcontres 31, 98166 Messina, Italy

E-mail: ebarbera@unime.it

F. Brini (Corresponding author)

Department of Mathematics and Alma Mater Research Center on Applied Mathematics

University of Bologna, via Saragozza 8, 40123 Bologna, Italy

E-mail: francesca.brini@unibo.it 
with respect to Classical Thermodynamics (CT). In fact, RET considers as field variables not only those of CT (mass density, momentum and energy) but also the stress tensor, the heat flux and others. The corresponding field equations are balance laws supplemented by local and instantaneous constitutive equations, that are determined by the requirement of validity of universal physical principles, like the entropy principle (existence of an entropy inequality and concavity of the entropy density) and the principle of relativity. During the last decades, RET proved to be a very powerful theory, capable of describing non-stationary physical phenomena through hyperbolic PDE systems, overcoming the paradox of infinite velocities due to parabolic PDE models. At the beginning, the theory was proposed by Müller, Ruggeri and other researchers for rarefied monatomic gases [4], but in the last years it has been generalized to rarefied polyatomic gases both in the classical [5] and in the relativistic framework [6] and also to quantum systems [7], obtaining relevant results and good agreement with experimental data.

In order to introduce a simple RET model for the metal electron gas, we will focus on the 8-moment model, that was already considered by Müller within the context of the kinetic theory [3,4]. Here we will follow a different macroscopic approach to write the equation system. However, a comparison with the model by Müller will be also presented and the advantages together with the limit of the present procedure of construction will be discussed. From another point of view, the mathematical peculiarities of the RET 8-moment models were summarized by Ruggeri in [8].

A very important question is related to the assignment of the boundary data when the electron gas is confined in a bounded domain, which is, of course, a very natural problem for the physical phenomena we are dealing with. Here, the boundary data prescription will be analyzed both from a macroscopic and a microscopic point of view, presenting, for the first time, the boundary conditions that have to be imposed to account for the interaction between the electrons and the boundaries.

\section{Balance equations}

In order to derive the balance equations, following $[1,2]$, we assume that the state of the electron gas is described by the phase density $f(\mathbf{x}, \mathbf{c}, t)$ of electrons at the position $\mathbf{x}$, with the velocity $\mathbf{c}$ and at the time $t$. The phase density must satisfy the Boltzmann equation, which assumes the form

$$
\frac{\partial f}{\partial t}+c_{i} \frac{\partial f}{\partial x_{i}}+f_{i} \frac{\partial f}{\partial c_{i}}=S,
$$

where $S$ stands for the collision term, while $f_{i}$ represents the specific external force acting on the particles which, in a metal gas, is the Lorentz force

$$
f_{i}=-\frac{q}{m}\left[E_{i}+(\mathbf{c} \wedge \mathbf{B})_{i}\right],
$$

with $-q$ the charge of an electron, $m$ its mass, ' $\wedge$ ' the symbol of the vector product, $\mathbf{E}$ the electromotive intensity and $\mathbf{B}$ the magnetic flux density. 
The multiplication of equations (1) by a generic function $\varphi(\mathbf{x}, \mathbf{c}, t)$ and the subsequent integration over the whole range of $\mathbf{c}$ provides the generic moment equation for the electron gas that is

$$
\frac{\partial \bar{\varphi}}{\partial t}+\frac{\partial \overline{c_{i} \varphi}}{\partial x_{i}}-\overline{\frac{\partial \varphi}{\partial t}+c_{i} \frac{\partial \varphi}{\partial x_{i}}+\left(f_{i}+i_{i}^{c}\right) \frac{\partial f}{\partial c_{i}}}=\int \varphi S \mathrm{~d} \mathbf{c} .
$$

The symbol $\bar{\varphi}$ denotes the moment $\int \varphi f \mathrm{~d} \mathbf{c}=\int_{-\infty}^{\infty} \int_{-\infty}^{\infty} \int_{-\infty}^{+\infty} \varphi f \mathrm{~d} c_{1} \mathrm{~d} c_{2} \mathrm{~d} c_{3}$.

In order to construct our macroscopic equation model, setting $\varphi=m, m c_{i}$, $m c^{2}, m c^{2} c_{i}, \ldots$ in equation (3), it is possible to construct an infinite hierarchy of moment equations that we truncate to the first eight moments:

$$
\begin{array}{ll}
\text { the mass density } & \rho=\int m f \mathrm{~d} \mathbf{c}, \\
\text { the mass flux or the momentum density } & J_{i}=\int m c_{i} f \mathrm{~d} \mathbf{c}, \\
\text { the energy density } & e=\frac{1}{2} \int m c^{2} f \mathrm{~d} \mathbf{c}, \\
\text { the heat flux } & q_{i}=\frac{1}{2} \int m c^{2} c_{i} f \mathrm{~d} \mathbf{c} .
\end{array}
$$

We also recall that $S_{i}=-\frac{q}{m} J_{i}$ is the electric current density.

From equation (3), we get the set of eight balance equations for the eight field variables $\rho, J_{i}, e$ and $q_{i}$ which assumes the form

$$
\begin{aligned}
& \frac{\partial \rho}{\partial t}+\frac{\partial J_{k}}{\partial x_{k}}=0, \\
& \frac{\partial J_{i}}{\partial t}+\frac{\partial P_{i k}}{\partial x_{k}}-\left(-\frac{q}{m} E_{i}\right) \rho-\left(-\frac{q}{m} \epsilon_{i j k} B_{k}\right) J_{j}=G_{i}, \\
& \frac{\partial e}{\partial t}+\frac{\partial q_{k}}{\partial x_{k}}-\left(-\frac{q}{m} E_{k}\right) J_{k}=0, \\
& \frac{\partial q_{i}}{\partial t}+\frac{1}{2} \frac{\partial \rho_{l l i k}}{\partial x_{k}}-\left(-\frac{q}{m} E_{k}\right)\left(P_{<i k>}+\frac{5}{3} e \delta_{i k}\right)-\left(-\frac{q}{m} \epsilon_{i j k} B_{k}\right) q_{j}=H_{i} .
\end{aligned}
$$

In the previous equations, $\epsilon_{i j k}$ is the Levi-Civita tensor, $P_{i j}=\int m c_{i} c_{j} f \mathrm{~d} \mathbf{c}$ represents the momentum flux, $\rho_{l l i k}=\int m c^{2} c_{i} c_{k} f \mathrm{~d} \mathbf{c}$ denotes the trace of the fourth moment, $\delta_{i k}$ the Kronecker tensor, $G_{i}$ and $H_{i}$ the productions, while the square brackets stand for the traceless part of a symmetric tensor.

In order to obtain a closed set of field equations from system (5), the fluxes $P_{<i k>}$ and $\rho_{l l i k}$ and the productions $G_{i}$ and $H_{i}$ must be expressed in terms of the eight field variables $\rho, J_{i}, e$ and $q_{i}$, by material dependent relations.

First of all, in the spirit of RET, we assume that these relations are local in space and time, so that at the time $t$ and in the position $\mathbf{x}$, they depend on the field at the same time and position, that is

$$
\begin{aligned}
& P_{<i j>}=P_{<i j>}\left(\rho, J_{i}, e, q_{i}\right), \\
& \rho_{l l i k}=\rho_{l l i k}\left(\rho, J_{i}, e, q_{i}\right), \\
& G_{i}=G_{i}\left(\rho, J_{i}, e, q_{i}\right) \\
& H_{i}=H_{i}\left(\rho, J_{i}, e, q_{i}\right) .
\end{aligned}
$$

Then, there are different ways to close the truncated moment system (see for example $[4,5]$ ) referring to the kinetic theory through a microscopic approach or following a macroscopic approach, based on the validity requirement 
of physics principle. Here, as already said in the introduction, we will close the system at a macroscopic level.

\section{The closure of the equation system}

\subsection{The entropy principle}

The entropy principle asserts the existence of a concave entropy density $h$, an entropy flux $\phi_{i}$ and a non-negative entropy production $\Sigma$, such that the balance equation

$$
\frac{\partial h}{\partial t}+\frac{\partial \phi_{i}}{\partial x_{i}}=\Sigma \geq 0
$$

holds for every thermodynamic process in an electron gas, that is to say for every solution of the field equation. Also the entropy quantities must be expressed in terms of the field variables by constitutive relations as (6), i.e.

$$
\begin{aligned}
& h=h\left(\rho, J_{i}, e, q_{i}\right), \\
& \phi_{i}=\phi_{i}\left(\rho, J_{i}, e, q_{i}\right) .
\end{aligned}
$$

In this section, we will refer to the RET procedure [4] and we will use the entropy principle in order to restrict the functional form of the constitutive functions (6) and (8):

The key of the evaluation of the entropy principle is that the entropy inequality (7) must hold for every thermodynamic process, that is for every solution of the balance equations (5). In this way, equations (5) can be considered as constrains for the fields to satisfy the entropy inequality and these constrains can be taken into account using the Lagrange multipliers, known as main field variables $[9,10]$. The main field components play a very relevant role: if we refer to them as independent field variables, the PDE system becomes symmetric hyperbolic and this fact guarantees the best mathematical properties of the solutions (see [5] for a historical review of this subject). Here, we assume the existence of eight main field components $\Lambda, \Gamma_{i}, \Psi$ and $\Pi_{i}$ that can be expressed in terms of the field variables through constitutive functions of the form (6), so that the inequality

$$
\begin{aligned}
\frac{\partial h}{\partial t}+\frac{\partial \phi_{i}}{\partial x_{i}} & -\Lambda\left[\frac{\partial \rho}{\partial t}+\frac{\partial J_{k}}{\partial x_{k}}\right] \\
& -\Gamma_{i}\left[\frac{\partial J_{i}}{\partial t}+\frac{\partial P_{i k}}{\partial x_{k}}-g_{i} \rho-l_{i j} J_{j}-G_{i}\right] \\
& -\Psi\left[\frac{\partial e}{\partial t}+\frac{\partial q_{k}}{\partial x_{k}}-g_{k} J_{k}\right] \\
& -\Pi_{i}\left[\frac{\partial q_{i}}{\partial t}+\frac{1}{2} \frac{\partial \rho_{l l i k}}{\partial x_{k}}-g_{k}\left(P_{<i k>}+\frac{5}{3} e \delta_{i k}\right)-l_{i k} q_{k}-H_{i}\right] \geq 0,
\end{aligned}
$$

must be valid for all $\rho, J_{i}, e$ and $q_{i}$. 
For the sake of simplicity, in equation (9) we have set

$$
g_{i}=-\frac{q}{m} E_{i} \quad \text { and } \quad l_{i j}=-\frac{q}{m} \epsilon_{i j k} B_{k} .
$$

Inserting the constitutive relations $(6,8)$ into inequality $(9)$, one obtains an expression which is linear in the derivatives of $\rho, J_{i}, e$ and $q_{i}$ with respect to $t$ and $x_{k}$. Then, since the inequality (9) must hold for every field variable, it holds also for every derivative of these quantities. Therefore, imposing that the coefficients of the derivatives vanish, one gets the following set of equations for the entropy density

$$
\frac{\partial h}{\partial \rho}=\Lambda, \quad \frac{\partial h}{\partial J_{i}}=\Gamma_{i}, \quad \frac{\partial h}{\partial e}=\Psi, \quad \frac{\partial h}{\partial q_{i}}=\Pi_{i},
$$

and the the entropy flux

$$
\begin{aligned}
& \frac{\partial \phi_{k}}{\partial \rho}=\Gamma_{i} \frac{\partial P_{<i k>}}{\partial \rho}+\frac{1}{2} \Pi_{i} \frac{\partial \rho_{l l i k}}{\partial \rho} \\
& \frac{\partial \phi_{k}}{\partial J_{i}}=\Gamma_{j} \frac{\partial P_{<j k>}}{\partial J_{i}}+\frac{1}{2} \Pi_{j} \frac{\partial \rho_{l l j k}}{\partial J_{i}}+\Lambda \delta_{i k}, \\
& \frac{\partial \phi_{k}}{\partial e}=\frac{2}{3} \Gamma_{k}+\Gamma_{i} \frac{\partial P_{<i k>}}{\partial e}+\frac{1}{2} \Pi_{i} \frac{\partial \rho_{l l i k}}{\partial e} \\
& \frac{\partial \phi_{k}}{\partial q_{i}}=\Gamma_{j} \frac{\partial P_{<j k>}}{\partial q_{i}}+\frac{1}{2} \Pi_{j} \frac{\partial \rho_{l l j k}}{\partial q_{i}}+\Psi \delta_{i k},
\end{aligned}
$$

while the residual inequality reads

$\Sigma=\Gamma_{i}\left[g_{i} \rho+l_{i j} J_{j}+G_{i}\right]+\Psi g_{k} J_{k}+\Pi_{i}\left[g_{k}\left(P_{<i k>}+\frac{5}{3} e \delta_{i k}\right)+l_{i j} q_{j}+H_{i}\right] \geq 0$.

Relations (11-13) summarizes all restrictions for the constitutive functions that can be derived from the entropy inequality.

\subsection{Approximation in the neighborhood of the equilibrium}

Relations (11-13) cannot be easily used in order to determine the required constitutive functions, for this reason we restrict our attention to processes not far away from the equilibrium state, characterized by vanishing fluxes $J_{i}$ and $q_{i}$. In this way, it is reasonable to approximate the constitutive functions for the entropy quantities, the fluxes and the main field components as

$$
\begin{aligned}
& h=h_{\mathrm{E}}(\rho, e)+h_{1}(\rho, e) J^{2}+h_{2}(\rho, e) J_{l} q_{l}+h_{3}(\rho, e) q^{2}, \\
& \phi_{i}=\phi_{1}(\rho, e) J_{i}+\phi_{2}(\rho, e) q_{i}, \\
& P_{<i j>}=0, \\
& \rho_{l l i j}=a(\rho, e) \delta_{i j}, \\
& \Lambda=\Lambda_{\mathrm{E}}(\rho, e)+\Lambda_{1}(\rho, e) J^{2}+\Lambda_{2}(\rho, e) J_{l} q_{l}+\Lambda_{3}(\rho, e) q^{2}, \\
& \Gamma_{i}=\Gamma_{1}(\rho, e) J_{i}+\Gamma_{2}(\rho, e) q_{i}, \\
& \Psi=\Psi_{\mathrm{E}}(\rho, e)+\Psi_{1}(\rho, e) J^{2}+\Psi_{2}(\rho, e) J_{l} q_{l}+\Psi_{3}(\rho, e) q^{2}, \\
& \Pi_{i}=\Pi_{1}(\rho, e) J_{i}+\Pi_{2}(\rho, e) q_{i},
\end{aligned}
$$


where the subscript "E" denotes the equilibrium quantity. We recall that a linearization of the constitutive expression for the fluxes requires that for the entropy quantities and the main field components also the quadratic terms are taken into account [4].

The assumptions (14) and the relations $(11,12)$ yield the following set of equations

$$
\begin{array}{llll}
\frac{\partial h_{\mathrm{E}}}{\partial \rho}=\Lambda_{\mathrm{E}}, & \frac{\partial h_{\mathrm{E}}}{\partial e}=\Psi_{\mathrm{E}}, & \\
\frac{\partial h_{1}}{\partial \rho}=\Lambda_{1}, & \frac{\partial h_{2}}{\partial \rho}=\Lambda_{2}, & & \frac{\partial h_{3}}{\partial \rho}=\Lambda_{3}, \\
\frac{\partial h_{1}}{\partial e}=\Psi_{1}, & \frac{\partial h_{2}}{\partial e}=\Psi_{2}, & \frac{\partial h_{3}}{\partial e}=\Psi_{3}, \\
2 h_{1}=\Gamma_{1}, & h_{2}=\Gamma_{2}=\Pi_{1}, & 2 h_{3}=\Pi_{2}, \\
\frac{\partial \phi_{1}}{\partial \rho}=\frac{1}{2} \Pi_{1} \frac{\partial a}{\partial \rho}, & \frac{\partial \phi_{2}}{\partial \rho}=\frac{1}{2} \Pi_{2} \frac{\partial a}{\partial \rho}, & \\
\frac{\partial \phi_{1}}{\partial e}=\frac{1}{2} \Pi_{1} \frac{\partial a}{\partial e}+\frac{2}{3} \Gamma_{1}, & \frac{\partial \phi_{2}}{\partial e}=\frac{1}{2} \Pi_{2} \frac{\partial a}{\partial e}+\frac{2}{3} \Gamma_{2}, & \\
\phi_{1}=\Lambda_{\mathrm{E}}, & \phi_{2}=\Psi_{\mathrm{E}} . &
\end{array}
$$

that must be exploited. We start considering the relations $(15)_{1,2}$ and, referring to them, we can deduce that

$$
\mathrm{dh}_{\mathrm{E}}=\Lambda_{\mathrm{E}} \mathrm{d} \rho+\Psi_{\mathrm{E}} \mathrm{de}
$$

Comparing (16) and the Gibbs relation

$$
\mathrm{dh}_{\mathrm{E}}=-\frac{\mathrm{g}}{\mathrm{T}} \mathrm{d} \rho+\frac{1}{\mathrm{~T}} \mathrm{de}
$$

it is possible to obtain the expression of $\Lambda$ and $\Psi$ at equilibrium:

$$
\Lambda_{\mathrm{E}}=-\frac{g}{T} \quad \text { and } \quad \Psi_{\mathrm{E}}=\frac{1}{T},
$$

where $T$ denotes the absolute temperature, $g=\frac{e}{\rho}-\frac{T h_{\mathrm{E}}}{\rho}+\frac{p_{\mathrm{E}}}{\rho}$ is the specific free enthalpy and $p_{\mathrm{E}}$ represents the equilibrium pressure.

Through some rearrangements of relations (15), it is possible to write a partial differential equation for $a$ as a function of $\rho$ and $e$ :

$$
\frac{\partial^{2} h_{\mathrm{E}}}{\partial e^{2}} \frac{\partial a}{\partial \rho}-\frac{\partial^{2} h_{\mathrm{E}}}{\partial \rho \partial e} \frac{\partial a}{\partial e}=\frac{4}{3} \frac{\partial^{2} h_{\mathrm{E}}}{\partial \rho^{2}} .
$$

It is easily verified that, if the expression of the entropy density at equilibrium $h_{\mathrm{E}}$ is known and equation (19) is integrated, all the remaining constitutive relations are determined explicitly from (15). Indeed, in terms of $h_{\mathrm{E}}$ and $a$, we get

$$
\begin{array}{lll}
\Lambda_{\mathrm{E}}=\phi_{1}=\frac{\partial h_{\mathrm{E}}}{\partial \rho}, & \Psi_{\mathrm{E}}=\phi_{2}=\frac{\partial h_{\mathrm{E}}}{\partial e}, & \\
\Pi_{1}=h_{2}=\Gamma_{2}=\frac{\partial \Lambda_{\mathrm{E}}}{\frac{\partial \rho}{2} \frac{\partial a}{\partial \rho}}, & \Gamma_{1}=2 h_{1}=\frac{3}{2}\left[\frac{\partial \Lambda_{\mathrm{E}}}{\partial e}-\frac{\frac{\partial \Lambda_{\mathrm{E}}}{\partial \rho}}{\frac{\partial a}{\partial \rho}} \frac{\partial a}{\partial e}\right], & \Pi_{2}=2 h_{3}=\frac{\partial \Psi_{\mathrm{E}}}{\frac{\partial \rho}{2} \frac{\partial a}{\partial \rho}}, \\
\Lambda_{1}=\frac{\partial h_{1}}{\partial \rho}, & \Lambda_{2}=\frac{\partial h_{2}}{\partial \rho}, & \Lambda_{3}=\frac{\partial h_{3}}{\partial \rho}, \\
\Psi_{1}=\frac{\partial h_{1}}{\partial e}, & \Psi_{2}=\frac{\partial h_{2}}{\partial e}, & \Psi_{3}=\frac{\partial h_{3}}{\partial e} .
\end{array}
$$




\subsection{Change of the independent variables}

To simplify the integration of equation (19), we change the independent variables from $\rho$ and $e$ to $-\frac{g}{T}$ and $\frac{1}{T}$ as usually done in RET [4]. So, from the Gibbs relation (17) and the Legendre transformation $h_{\mathrm{E}}=\frac{p_{\mathrm{E}}}{T}-\frac{g}{T} \rho+\frac{1}{T} e$, we get

$$
\mathrm{d}\left(\frac{\mathrm{p}_{\mathrm{E}}}{\mathrm{T}}\right)=-\mathrm{ed}\left(\frac{1}{\mathrm{~T}}\right)-\rho \mathrm{d}\left(-\frac{\mathrm{g}}{\mathrm{T}}\right)
$$

where $p_{\mathrm{E}}=p_{\mathrm{E}}\left(\frac{1}{T},-\frac{g}{T}\right)$, and

$$
e\left(\frac{1}{T},-\frac{g}{T}\right)=-p_{\mathrm{E}}-\frac{1}{T}\left(\frac{\partial p_{\mathrm{E}}}{\partial\left(\frac{1}{T}\right)}\right)_{\frac{g}{T}}, \quad \rho\left(\frac{1}{T},-\frac{g}{T}\right)=-\frac{1}{T}\left(\frac{\partial p_{\mathrm{E}}}{\partial\left(-\frac{g}{T}\right)}\right)_{T} .
$$

The subscribed symbols denote that the indicated quantities are maintained constants during the derivations.

After some calculations, equation (19) can be rewritten in terms of the new independent variables as

$$
\frac{\partial a}{\partial\left(-\frac{g}{T}\right)}=-\frac{4}{3}\left[2 \frac{\partial p_{\mathrm{E}}}{\partial\left(\frac{1}{T}\right)}+\frac{1}{T} \frac{\partial^{2} p_{\mathrm{E}}}{\partial\left(\frac{1}{T}\right)^{2}}\right]
$$

Furthermore, since we are dealing with a monatomic gas, we have $p_{\mathrm{E}}=\frac{2}{3} e$ so from equation $(22)_{1}$ we get

$$
p_{\mathrm{E}}\left(\frac{1}{T},-\frac{g}{T}\right)=T^{\frac{5}{2}} F\left(-\frac{g}{T}\right)
$$

The previous relation expresses the thermal equation of state as a product of functions depending on a single independent variable. Consequently, from (22) and integrating (23) we can write

$$
\begin{aligned}
& e\left(\frac{1}{T},-\frac{g}{T}\right)=\frac{3}{2} T^{\frac{5}{2}} F\left(-\frac{g}{T}\right), \\
& \rho\left(\frac{1}{T},-\frac{g}{T}\right)=-T^{\frac{3}{2}} F^{\prime}\left(-\frac{g}{T}\right), \\
& a\left(\frac{1}{T},-\frac{g}{T}\right)=-5 T^{\frac{7}{2}} \int F\left(-\frac{g}{T}\right) \mathrm{d}\left(-\frac{g}{T}\right)+K\left(\frac{1}{T}\right) .
\end{aligned}
$$

The problem of the determinations of the constitutive functions in the neighborhood of equilibrium is reduced to the determinations of the two singlevariable functions $F\left(-\frac{g}{T}\right)$ and $K\left(\frac{1}{T}\right)$. Once we know them, the complete set of field equations appropriate to this process can be recovered from (20). 


\subsection{Determination of the unknown functions}

The unknown function $F\left(-\frac{g}{T}\right)$ can be determined referring to statistical thermodynamics. In fact, as it is well-known, electrons are fermions and their corresponding equilibrium distribution function reads

$$
f_{\mathrm{E}}=\frac{y}{e^{-\frac{m}{k_{B}} \frac{g}{T}+\frac{m c^{2}}{2 k_{B}} \frac{1}{T}}+1}=\frac{y}{e^{-\frac{m}{k_{B}} \frac{g}{T}+\frac{m c^{2}}{2 k_{B}} \frac{1}{T}}+1},
$$

where $k_{B}$ denotes the Boltzmann constant. From $p_{\mathrm{E}}=\frac{1}{3} \int m c^{2} f_{\mathrm{E}} \mathrm{d} \mathbf{c}$ and (26), we obtain the explicit expression for the equilibrium pressure that is

$$
p_{\mathrm{E}}=\frac{4}{3} \pi m y\left(2 \frac{k_{B}}{m} T\right)^{\frac{5}{2}} i_{4}(\alpha)
$$

with

$$
\alpha=-\frac{m}{k_{B}} \frac{g}{T} \quad \text { and } \quad i_{n}(\alpha)=\int_{0}^{+\infty} \frac{x^{n}}{e^{\alpha+x^{2}+1}} \mathrm{~d} x .
$$

The integral function $i_{n}(\alpha)$ is well-known in the framework of degenerate gases theory and several techniques have been developed to calculate it numerically, for an exhaustive review see [11]. Such a function presents some peculiar properties, we recall, among the others, the following recurrence relation

$$
\frac{\mathrm{di}_{\mathrm{n}}(\alpha)}{\mathrm{d} \alpha}=-\frac{n-1}{2} i_{n-2}(\alpha)
$$

Now, from comparison of (27) and (24), it is possible to get the explicit form of the function $F\left(-\frac{g}{T}\right)$, that is

$$
F\left(-\frac{g}{T}\right)=\frac{4}{3} \pi m y\left(2 \frac{k_{B}}{m}\right)^{\frac{5}{2}} i_{4}(\alpha)
$$

and, from (25) and (29), we also get

$$
\begin{aligned}
& e\left(\frac{1}{T},-\frac{g}{T}\right)=2 \pi m y\left(2 \frac{k_{B}}{m} T\right)^{\frac{5}{2}} i_{4}(\alpha), \\
& \rho\left(\frac{1}{T},-\frac{g}{T}\right)=4 \pi m y\left(2 \frac{k_{B}}{m} T\right)^{\frac{3}{2}} i_{2}(\alpha), \\
& a\left(\frac{1}{T},-\frac{g}{T}\right)=\frac{4}{3} \pi m y\left(2 \frac{k_{B}}{m} T\right)^{\frac{7}{2}} i_{6}(\alpha)+K\left(\frac{1}{T}\right) .
\end{aligned}
$$

The remaining unknown function $K\left(\frac{1}{T}\right)$ cannot be determined through a macroscopic closure procedure. Hence, we conclude that our macroscopic approach furnishes a family of PDE systems that contain an arbitrary function $K(1 / T)$. Such a function can be fixed only phenomenologically by a comparison with some physical results or microscopically by a comparison with the results of kinetic theroy. 


\subsection{Concavity of the entropy density}

Here we analyze the consequences of the concavity requirement, a condition which follows directly from the entropy principle and that must hold also for the present equation system. Since $h$ must be a concave function of its arguments, at least in the neighborhood of the equilibrium state, we have to impose that the Jacobian of $h_{\mathrm{E}}$

$$
\left\|\begin{array}{cc}
-\frac{\partial^{2} h_{\mathrm{E}}}{\partial \rho^{2}} & -\frac{\partial^{2} h_{\mathrm{E}}}{\partial \rho \partial e} \\
-\frac{\partial^{2} h_{\mathrm{E}}}{\partial \rho \partial e} & -\frac{\partial^{2} h_{\mathrm{E}}}{\partial e^{2}}
\end{array}\right\|
$$

is a positive-definite matrix.

Taking into account expressions (25) and the change of the independent variables described in the subsection 3.3, the requirement of concavity at equilibrium implies the following conditions for the function $F(\alpha)$ :

$$
F>0, \quad F^{\prime \prime}>0, \quad 5 F F^{\prime \prime}>3 F^{2} .
$$

It is possible to prove the following theorem:

Theorem 1 The equation system (5) is equipped with an entropy law with concave entropy density in the neighborhood of equilibrium, if the gas is not completely degenerate.

Proof 1 The proof is available in Appendix A.

\subsection{Summary of the results}

Here, we briefly summarize the results concerning the constitutive relations, obtained in the previous subsections. Explicitly the field equations are

$$
\begin{aligned}
& \frac{\partial \rho}{\partial t}+\frac{\partial J_{k}}{\partial x_{k}}=0, \\
& \frac{\partial J_{i}}{\partial t}+\frac{2}{3} \frac{\partial e}{\partial x_{i}}-g_{i} \rho-l_{i j} J_{j}=G_{i} \\
& \frac{\partial e}{\partial t}+\frac{\partial q_{k}}{\partial x_{k}}-g_{k} J_{k}=0, \\
& \frac{\partial q_{i}}{\partial t}+\frac{1}{2} \frac{\partial a}{\partial x_{i}}-\frac{5}{3} e g_{i}-l_{i j} q_{j}=H_{i},
\end{aligned}
$$

while the entropy quantities and the main field components read

$$
\begin{aligned}
& h=\frac{4}{3} \pi m y \theta^{\frac{3}{2}}\left[\frac{k}{m} I_{4}(\alpha)-\frac{g}{T} I_{2}(\alpha)\right]+h_{1} J^{2}+h_{2} J_{l} q_{l}+h_{3} q^{2}, \\
& \phi_{i}=\frac{1}{T} q_{i}-\frac{g}{T} J_{i} \\
& \Lambda=-\frac{g}{T}+\Lambda_{1}(\rho, e) J^{2}+\Lambda_{2}(\rho, e) J_{l} q_{l}+\Lambda_{3}(\rho, e) q^{2}, \\
& \Gamma_{i}=\Gamma_{1} J_{i}+\Gamma_{2} q_{i}, \\
& \Psi=\frac{1}{T}+\Psi_{1}(\rho, e) J^{2}+\Psi_{2}(\rho, e) J_{l} q_{l}+\Psi_{3}(\rho, e) q^{2} \\
& \Pi_{i}=\Pi_{1} J_{i}+\Pi_{2} q_{i} .
\end{aligned}
$$


where

$$
\begin{aligned}
& \Pi_{1}=h_{2}=\Gamma_{2}=3 \beta \theta^{-\frac{7}{2}} \frac{I_{4}}{I_{2} I_{6}-I_{4}^{2}}, \\
& \Pi_{2}=2 h_{3}=-6 \beta \theta^{-\frac{9}{2}} \frac{I_{2}}{I_{2} I_{6}-I_{4}^{2}}, \\
& \Gamma_{1}=2 h_{1}=-\frac{3}{2} \beta \theta^{-\frac{5}{2}} \frac{I_{6}}{I_{2} I_{6}-I_{4}^{2}}, \\
& \Lambda_{1}=\frac{3}{16} \frac{m}{k_{B}} \beta^{2} \theta^{-4} \frac{7 I_{4}^{4}-5 I_{2} I_{4}^{2} I_{6}-5 I_{2}^{2} I_{6}^{2}+3 I_{0} I_{4} I_{6}^{2}}{\left(I_{2} I_{6}-I_{4}^{2}\right)^{2}\left(I_{0} I_{4}-I_{2}^{2}\right)}, \\
& \Lambda_{2}=-\frac{9}{4} \frac{m}{k_{B}} \beta^{2} \theta^{-5} \frac{3 I_{2} I_{4}^{2}-4 I_{2}^{2} I_{6}+I_{0} I_{4} I_{6}}{\left(I_{2} I_{6}-I_{4}^{2}\right)^{2}\left(I_{0} I_{4}-I_{2}^{2}\right)} I_{4}, \\
& \Lambda_{3}=\frac{9}{4} \frac{m}{k_{B}} \beta^{2} \theta^{-6} \frac{2 I_{2}^{2} I_{4}^{2}+I_{0} I_{4}^{3}-3 I_{2}^{3} I_{6}}{\left(I_{2} I_{6}-I_{4}^{2}\right)^{2}\left(I_{0} I_{4}-I_{2}^{2}\right)}, \\
& \Psi_{1}=-\frac{3}{8} \frac{m}{k_{B}} \beta^{2} \theta^{-5} \frac{7 I_{2} I_{4}^{3}-10 I_{2}^{2} I_{4} I_{6}+5 I_{0} I_{4}^{2} I_{6}-2 I_{0} I_{2} I_{6}^{2}}{\left(I_{2} I_{6}-I_{4}^{2}\right)^{2}\left(I_{0} I_{4}-I_{2}^{2}\right)} \\
& \Psi_{2}=\frac{3}{2} \frac{m}{k_{B}} \beta^{2} \theta^{-6} \frac{2 I_{2}^{2} I_{4}^{2}+7 I_{0} I_{4}^{3}-5 I_{2}^{3} I_{6}-4 I_{0} I_{2} I_{4} I_{6}}{\left(I_{2} I_{6}-I_{4}^{2}\right)^{2}\left(I_{0} I_{4}-I_{2}^{2}\right)}, \\
& \Psi_{3}=\frac{9}{2} \frac{m}{k_{B}} \beta^{2} \theta^{-7} \frac{I_{2}^{2} I_{4}-4 I_{0} I_{4}^{2}-5 I_{2}^{3} I_{6}+3 I_{0} I_{2} I_{6}}{\left(I_{2} I_{6}-I_{4}^{2}\right)^{2}\left(I_{0} I_{4}-I_{2}^{2}\right)},
\end{aligned}
$$

with the following notations

$$
\theta=2 \frac{k_{B}}{m} T, \quad \beta=\frac{1}{\pi y m^{2}}, \quad I_{n}(\alpha)=(n+1) i_{n}(\alpha) .
$$

The PDE system can be also rewritten focusing on the eight new independent field variables $\left(\alpha, J_{i}, T, q_{i}\right)$. Indeed, from the previous relation, it is easily proven that

$$
\begin{array}{ll}
\rho_{T}=4 k_{B} \pi y \theta^{\frac{1}{2}} I_{2}(\alpha), & \rho_{\alpha}=-2 \pi m y \theta^{\frac{3}{2}} I_{0}(\alpha), \\
e_{T}=2 k_{B} \pi y \theta^{\frac{3}{2}} I_{4}(\alpha), & e_{\alpha}=-\pi m y \theta^{\frac{5}{2}} I_{2}(\alpha), \\
a_{T}=\frac{4}{3} k_{B} \pi y \theta^{\frac{5}{2}}\left(I_{6}(\alpha)-\frac{\tilde{K}(1 / T)}{T^{2}}\right), & a_{\alpha}=-\frac{2}{3} \pi m y \theta^{\frac{7}{2}} I_{4}(\alpha),
\end{array}
$$

with $K(1 / T)=\frac{4}{3} k_{B} \pi y \theta^{\frac{5}{2}} \tilde{K}(1 / T)$. Then, taking into account such relations, the model equation can be also rewritten as

$$
\begin{aligned}
& 4 k_{B} \pi y \theta^{\frac{1}{2}} I_{2}(\alpha) \frac{\partial T}{\partial t}-2 \pi m y \theta^{\frac{3}{2}} I_{0}(\alpha) \frac{\partial \alpha}{\partial t}+\frac{\partial J_{k}}{\partial x_{k}}=0, \\
& \frac{\partial J_{i}}{\partial t}+\frac{4}{3} k_{B} \pi y \theta^{\frac{3}{2}} I_{4}(\alpha) \frac{\partial T}{\partial x_{i}}-\frac{2}{3} \pi m y \theta^{\frac{5}{2}} I_{2}(\alpha)\left[\frac{\partial \alpha}{\partial x_{i}}+\frac{1}{\frac{k_{B}}{m} T} g_{i}\right]-l_{i j} J_{j}=G_{i}, \\
& 2 k_{B} \pi y \theta^{\frac{3}{2}} I_{4}(\alpha) \frac{\partial T}{\partial t}-\pi m y \theta^{\frac{5}{2}} I_{2}(\alpha) \frac{\partial \alpha}{\partial t}+\frac{\partial q_{k}}{\partial x_{k}}-g_{k} J_{k}=0, \\
& \frac{\partial q_{i}}{\partial t}+\frac{2}{3} k_{B} \pi y \theta^{\frac{5}{2}}\left(I_{6}(\alpha)-\frac{\tilde{K}\left(T^{-1}\right)}{T^{2}}\right) \frac{\partial T}{\partial x_{i}}- \\
& -\frac{1}{3} \pi m y \theta^{\frac{7}{2}} I_{4}(\alpha)\left[\frac{\partial \alpha}{\partial x_{i}}+\frac{1}{\frac{k_{B}}{m} T} g_{i}\right] l_{i j} q_{j}=H_{i} .
\end{aligned}
$$




\subsection{Production terms}

The validity of the entropy principle involves also that the production terms $G_{i}$ and $H_{i}$ must satisfy the residual inequality (13). Referring to relations (35) and (36), one can easily verify that the residual inequality reduces to the following expression if terms of order higher than the second one are neglected:

$$
\Sigma=\Gamma_{i} G_{i}+\Pi_{i} H_{i} \geq 0 .
$$

Moreover, the production terms have to be linearized in the neighborhood of the equilibrium according to the approximation approach used in the previous sections. Starting from these two requirements, it is natural to assume that $\Sigma$ is a positive quadratic form in the main field components $\Gamma_{i}$ and $\Pi_{i}$. Hence, if we denote by $\boldsymbol{\Gamma}=\left(\Gamma_{1}, \Gamma_{2}, \Gamma_{3}\right)$ and $\boldsymbol{\Pi}=\left(\Pi_{1}, \Pi_{2}, \Pi_{3}\right)$ and define $\mathbf{A}=\mathbf{A}(\alpha, 1 / T)$ as a $6 \times 6$ symmetric positive-definite matrix, that could possibly depend only on the equilibrium variables $\alpha$ and $1 / T$, we have

$$
\mathbf{w}=(\boldsymbol{\Gamma}, \boldsymbol{\Pi})^{T} \quad \Sigma=\mathbf{w}^{T} \mathbf{A} \mathbf{w} \geq 0 .
$$

This implies that the production terms are expressed as

$$
(\mathbf{G}, \mathbf{H})^{T}=\mathbf{A} \mathbf{w}
$$

and, since the main field components $\Gamma_{i}$ and $\Pi_{i}$ are expressed as linear combination of $J_{i}$ and $q_{i}$, it is easily verified that the production terms are also combination of $J_{i}$ and $q_{i}$.

In this case, as usual, we cannot determine the explicit expression of the matrix A referring only to the macroscopic principles. In fact, it is possible to go behind the generic properties of $\mathbf{A}$ only through a comparison with physical phenomena or with kinetic theory results. This fact is not surprising since at a macroscopic level we are not able to prescribe the nature of the interactions between particles: only the physical macroscopic effects can be added to a phenomenological model.

\section{A comparison with the kinetic theory closure}

As already said in the introduction, the RET 8-moment model was already derived by Müller $[3,4]$ through a microscopic closure. Such an approach is based on the expansion of the distribution function near equilibrium, obtaining a Grad-type expression [12] for $f=f_{\mathrm{E}}(T)+f^{(1)}\left(c_{1}, c_{2}, c_{3}\right)$

$$
f=\frac{y}{e^{-\frac{m}{k_{B}} \frac{g}{T}+\frac{m c^{2}}{2 k_{B}} \frac{1}{T}}+1}-\frac{y e^{-\frac{m}{k_{B}} \frac{g}{T}+\frac{m c^{2}}{2 k_{B}} \frac{1}{T}}}{\left(e^{-\frac{m}{k_{B}} \frac{g}{T}+\frac{m c^{2}}{2 k_{B}} \frac{1}{T}}+1\right)^{2}}\left(m c_{i} \Gamma_{i}+m c^{2} c_{i} \hat{\Pi}_{i}\right),
$$

where $\hat{\Pi}_{i}=\Pi_{i} / 2$, and thanks to (6), it is possible to write: 


$$
\left[\begin{array}{l}
J_{i} \\
2 q_{i}
\end{array}\right]=\left[\begin{array}{ll}
K_{2} & K_{4} \\
K_{4} & K_{6}
\end{array}\right]\left[\begin{array}{c}
\Gamma_{i} \\
\hat{\Pi}_{i}
\end{array}\right]
$$

with

$$
K_{A}=-\frac{4 \pi m^{2} y}{3} \int \frac{e^{-\frac{m}{k_{B}} \frac{g}{T}+\frac{m c^{2}}{2 k_{B}} \frac{1}{T}}}{\left(e^{-\frac{m}{k_{B}} \frac{g}{T}+\frac{m c^{2}}{2 k_{B}} \frac{1}{T}}+1\right)^{2}} c^{A} \mathrm{~d} c=-\frac{2}{3} \pi m^{2} y \theta^{\frac{A+3}{2}} I_{A}(\alpha) .
$$

Relation (43) can be inverted, obtaining the expressions for $\Gamma_{i}$ and $\Pi_{i}$ in accordance with (35) and (36). Carrying on the calculation, one obtains the same results for all the densities and the fluxes except for $a$. A rapid comparison between our results and those in $[3,4]$ shows that the closure at microscopic level implies that $K(1 / T)$, the arbitrary function of our model, must vanish.

The kinetic theory is able to furnish also an explicit expression of the production terms, indeed after some calculations $[3,4]$, the production terms read

$$
\left[\begin{array}{l}
G_{i} \\
2 H_{i}
\end{array}\right]=-\frac{1}{l}\left[\begin{array}{cc}
K_{3} & K_{5} \\
K_{5} & K_{7}
\end{array}\right]\left[\begin{array}{c}
\Gamma_{i} \\
\hat{\Pi}_{i}
\end{array}\right],
$$

where $l$ denotes the mean free path of an electron between two collisions.

These production terms can be seen as a special case of (41) and it is possible to show the the corresponding matrix $\mathbf{A}$ is positive definite (the proof is similar to the one in Appendix A).

In conclusion, the 8-moment equations that we have introduced in the previous sections can be seen as a family of PDE system that contains also the model from the kinetic theory.

\section{Hyperbolicity property}

In the previous sections we have approximated the constitutive functions in the neighborhood of an equilibrium state. Therefore we have obtained an equation system that could satisfy the hyperbolicity property characteristic of RET only in a neighborhood of the equilibrium. In this section we will check the validity of such a property. To this aim we recall that a PDE system in the vector form

$$
A^{0} \frac{\partial \mathbf{U}}{\partial t}+A^{i} \frac{\partial \mathbf{U}}{\partial x_{i}}=\mathbf{B}
$$

is defined hyperbolic in the t-direction if $\operatorname{det}\left(A^{0}\right) \neq 0$ and if the eigenvalue problem

$$
\operatorname{det}\left(A^{i} n_{i}-\lambda A^{0}\right)=0,
$$

admits only real eigenvalues $\lambda$ and a set of linearly independent right eigenvectors $\mathbf{d}$, for all unit vectors $\mathbf{n}$. 
In the present model, if we consider as field components $\mathbf{U}=\left(\alpha, J_{i}, T, q_{i}\right)^{T}$, we have

$$
\operatorname{det}\left(A^{0}\right)=\operatorname{det}\left[\begin{array}{cccc}
\rho_{\alpha} & 0 & \rho_{T} & 0 \\
0 & 1 & 0 & 0 \\
e_{\alpha} & 0 & e_{T} & 0 \\
0 & 0 & 0 & 1
\end{array}\right]=\rho_{\alpha} e_{T}-e_{\alpha} \rho_{T}
$$

which, taking into account $(31)$, yields $\operatorname{det}\left(A^{0}\right)=-4 \pi^{2} k_{B} m y^{2} \theta^{3}\left(I_{0} I_{4}-I_{2}^{2}\right) \neq$ 0 (see Appendix B).

The characteristic equation (47) reads

$$
\operatorname{det}\left[\begin{array}{cccc}
-\lambda \rho_{\alpha} & n_{i} & -\lambda \rho_{T} & 0 \\
\frac{2}{3} e_{\alpha} n_{i} & -\lambda & \frac{2}{3} e_{T} n_{i} & 0 \\
-\lambda e_{\alpha} & 0 & -\lambda e_{T} & n_{i} \\
\frac{1}{2} a_{\alpha} n_{i} & 0 & \frac{1}{2} a_{T} n_{i} & -\lambda
\end{array}\right]=0
$$

which becomes

$$
\left(\rho_{\alpha} e_{T}-e_{\alpha} \rho_{T}\right) \lambda^{4}+\frac{1}{2}\left(\rho_{T} a_{\alpha}-\rho_{\alpha} a_{T}\right) \lambda^{2}+\frac{1}{3}\left(e_{\alpha} a_{T}-e_{T} a_{\alpha}\right)=0 .
$$

When the function $K(1 / T)=0$, that is to say for the model obtained through a comparison with the kinetic theory, the characteristic polynomial reads:

$$
\left(I_{0} I_{4}-I_{2}^{2}\right)\left(\frac{\lambda}{\sqrt{2 \frac{k_{B}}{m} T}}\right)^{4}+\frac{1}{3}\left(I_{2} I_{4}-I_{0} I_{6}\right)\left(\frac{\lambda}{\sqrt{2 \frac{k_{B}}{m} T}}\right)^{2}+\frac{1}{9}\left(I_{2} I_{6}-I_{4}^{2}\right)=0 .
$$

We are dealing with a biquadratic equation and it is reasonable to conjecture (see Appendix B) that it presents two copule of real opposite eigenvalues unless the gas is completely degenerate. So, we can conclude that the approximated system is always hyperbolic.

\section{Boundary data assignment}

In RET the prescription of the boundary conditions represents often a hurdle, well-known in the literature and different methods have been proposed to prescribe the conditions in different frameworks (some examples can be found in $[13-16])$. The reason is easily understood, if we recall that the field variables in RET include stress tensor components or higher order moments whose values cannot be observed or fixed in an experiment.

If we consider the metal electrons confined in a bounded domain, we have to carefully analysis the questions related to assignment of the boundary data. In this section, we will focus on this problem, limiting our attention to the $1 \mathrm{D}$ case, that is to say we imagine the electrons confined in a wire, whose section is negligible with respect to its length. For time-dependent phenomena associated to this system we deal with 4 hyperbolic PDEs with 4 non-vanishing 
characteristic velocities, 2 positive and two negative ones (see Appendix B). Hence, we have to prescribe at each wire end two boundary data. A possibility is to assign at the ends of the wire the temperature values and the electric current density.

In the particular case of a stationary phenomenon, the number of boundary data required to solve the system of ODEs is the same as before, since it corresponds to the number of non-zero characteristic velocities. The stationary assumption implies that the electric current density is conserved and so an additional condition is required in order to deal with a well-posed stationary problem. However, in this case, the total number of electrons inside the wire is conserved and the electron number conservation represents the additional condition we are looking for. In other words, in the present case we do not deal with non-controllable boundary data, since the number of the physical quantities that can be prescribed at the boundary is exactly the same as the number of data that are required for the integration of the equation system. So, at a macroscopic level the problem of the boundary data assignment is completely solved.

Nevertheless, if we want to take into account the interaction effect between the electrons and the metal walls, we can refer to an approach similar to that used by Grad [12] in order to describe the interaction between the molecules of a monatomic rarefied gas and the boundary. The idea (already known in kinetic theory for this case [17]) is to account simultaneously two different mechanisms: the specular reflection and the diffuse reflection. We recall that a particle is spectacularly reflected by the boundary if it is scattered elastically at the surface with reversal of the velocity component normal to the surface, while it is perfectly diffuse reflected if it is brought to thermal equilibrium at the surface.

To better illustrate the basis of the method, we consider a wire of length $L$ lying on the $x_{1}$ axis, so that its ends are at $x_{1}=0$ and $x_{1}=L$. As an example, we just focus on the boundary at $x_{1}=0$. We denote the incident distribution function as $f^{-}\left(c_{1}, c_{2}, c_{3}\right)$, so $f^{-}\left(c_{1}, c_{2}, c_{3}\right)=0$ if $c_{1}>0$, and the reflected distribution function by $f^{+}\left(c_{1}, c_{2}, c_{3}\right)$, where $f^{+}\left(c_{1}, c_{2}, c_{3}\right)=0$ if $c_{1}<0$. Therefore, the distribution function at the boundary reads:

$$
f\left(c_{1}, c_{2}, c_{3}\right)=f^{-}\left(c_{1}, c_{2}, c_{3}\right)+f^{+}\left(c_{1}, c_{2}, c_{3}\right) .
$$

We assume that the reflected function is expressed as a combination of specular and diffuse reflections:

$$
f^{+}\left(c_{1}, c_{2}, c_{3}\right)=p f^{-}\left(-c_{1}, c_{2}, c_{3}\right)+\mathcal{K} f_{\mathrm{E}}\left(T_{\mathrm{B}}\right),
$$

where $f_{\mathrm{E}}$ is the distribution function at equilibrium, $T_{\mathrm{B}}$ represents the temperature of the metal boundary, $\mathcal{K}$ is a suitable quantity that will be determined later and the parameter $p \in[0,1]$ represents the portion of electron spectacularly reflected by the wall.

Following Grad's approach, we firstly concentrate on the particular case $p=1$, that is to say the completely specular reflection, for such a case the 
distribution function at the boundary

$$
f\left(c_{1}, c_{2}, c_{3}\right)=f^{-}\left(c_{1}, c_{2}, c_{3}\right)+f^{-}\left(-c_{1}, c_{2} c_{3}\right)
$$

is even in $c_{1}$. Hence, it is possible to prescribe conditions for the moments corresponding to odd powers of $c_{1}$ that turn out to be always zero at the boundaries, while the moments corresponding to even powers of $c_{1}$ cannot be fixed automatically. In this way, the number of prescription at each boundary is equal to the number of moments corresponding to an odd power of $c_{1}$.

This last result is valid for completely specular reflection, but we want to extend here the method also for the cases $p<1$ and we take into account only the moments $J_{1}$ and $q_{1}$ (see (5)).

Let us consider the stationary case and $-q J_{1} / m$ denotes the constant value of the electric current density in $x_{1}=0$, we impose that

$$
\begin{aligned}
& J_{1}=\int m c_{1} f\left(c_{1}, c_{2}, c_{3}\right) \mathrm{d} \mathbf{c}= \\
& =\int_{c_{1}<0} m c_{1} f^{-}\left(c_{1}, c_{2}, c_{3}\right) \mathrm{d} \mathbf{c}+\int_{c_{1}>0} m c_{1} f^{+}\left(c_{1}, c_{2}, c_{3}\right) \mathrm{d} \mathbf{c}= \\
& =(p-1) \int_{c_{1}>0} m c_{1} f^{-}\left(-c_{1}, c_{2}, c_{3}\right) \mathrm{d} \mathbf{c}+\mathcal{K} \int_{c_{1}>0} m c_{1} f_{\mathrm{E}}\left(T_{\mathrm{B}}\right) \mathrm{d} \mathbf{c} .
\end{aligned}
$$

Recalling that in the present case the expression of the linearized distribution function for 1D systems is (42), the previous formula (55) is rewritten as

$$
\begin{array}{r}
J_{1}=\int_{c_{1}>0} m c_{1}\left[(p-1) f_{\mathrm{E}}(T)+\mathcal{K} f_{\mathrm{E}}\left(T_{\mathrm{B}}\right)\right] \mathrm{d} \mathbf{c}+ \\
\quad+(p-1) \int_{c_{1}>0} m c_{1} f^{(1)}\left(-c_{1}, c_{2}, c_{3}\right) \mathrm{d} \mathbf{c} .
\end{array}
$$

After some calculations (see Appendix C for details) we get

$$
\begin{aligned}
J_{1}= & m \pi y\left[\left((p-1) \theta^{2}+\mathcal{K} \theta_{B}^{2}\right) i_{3}(\alpha)+\right. \\
& \left.+(p-1) m\left(\Gamma_{1} \theta^{5 / 2} i_{2}(\alpha)+\frac{5}{3} \hat{\Pi}_{1} \theta^{7 / 2} i_{4}(\alpha)\right)\right]
\end{aligned}
$$

and from the previous formulas we can deduce $\mathcal{K}$ :

$$
\mathcal{K}=0 \quad \text { if } \quad c_{1}<0 \text { and } \mathcal{K}=(1-p) \frac{\theta^{2}}{\theta_{B}^{2}}+\frac{(p+1)}{2 m \pi y \theta_{B}^{2} i_{3}(\alpha)} J_{1} \quad \text { for } c_{1}>0 .
$$

Likewise, we write now the relation for $q_{1}$

$$
\begin{aligned}
& q_{1}=\frac{1}{2} \int m c^{2} c_{1} f \mathrm{~d} \mathbf{c}= \\
& =\int_{c_{1}<0} m c^{2} c_{1} f^{-}\left(c_{1}, c_{2}, c_{3}\right) \mathrm{d} \mathbf{c}+\int_{c_{1}>0} m c^{2} c_{1} f^{+}\left(c_{1}, c_{2}, c_{3}\right) \mathrm{d} \mathbf{c}= \\
& =(p-1) \int_{c_{1}>0} m c^{2} c_{1} f^{-}\left(-c_{1}, c_{2}, c_{3}\right) \mathrm{d} \mathbf{c}+\mathcal{K} \int_{c_{1}>0} m c^{2} c_{1} f_{\mathrm{E}}\left(T_{\mathrm{B}}\right) \mathrm{d} \mathbf{c} .
\end{aligned}
$$

Again, recalling formula (42), we have:

$$
\begin{array}{r}
q_{1}=\int_{c_{1}>0} m c^{2} c_{1}\left[(p-1) f_{\mathrm{E}}(T)+\mathcal{K} f_{\mathrm{E}}\left(T_{\mathrm{B}}\right)\right] \mathrm{d} \mathbf{c}+ \\
\quad+(p-1) \int_{c_{1}>0} m c^{2} c_{1} f^{(1)}\left(-c_{1}, c_{2}, c_{3}\right) \mathrm{d} \mathbf{c} .
\end{array}
$$


After some cumbersome calculations similar to the previous ones, we conclude that

$$
q_{1}=\frac{\pi m y}{6(1+p)}\left[(p-1) \theta^{3}+\mathcal{K} \theta_{B}^{3}\right] I_{5}(\alpha),
$$

which can be also rewritten as

$$
q_{1}=\frac{\pi m y}{6} \frac{1-p}{1+p} \theta^{2}\left(\theta_{B}-\theta\right) I_{5}(\alpha)+\frac{1}{3} \theta_{B} \frac{I_{5}(\alpha)}{I_{3}(\alpha)} J_{1} .
$$

This last equation gives the implicit relation between the temperature of the metal at the boundary and the electron temperature at the boundary that has to be used in the ODE integration.

Similar steps can be followed to obtain the boundary conditions at $x_{1}=L$.

\section{Appendix A}

\section{Proof of Theorem 1}

In subsection 3.5 we have already recalled that the concavity condition for our model at equilibrium reduces to the three conditions for $F(\alpha)$ given in (33). In what follows we prove that these conditions are verified if $F(\alpha)$ is the function in (30) except for a completely degenerate gas. Indeed, from (29) and (31), it is immediately verified that $F>0$ and $\frac{d^{2} F}{d \alpha^{2}}>0$. So, now we focus on the third condition $(33)_{3}$. Explicitly, we have

$$
5 F F^{\prime \prime}-3\left(F^{\prime}\right)^{2}=\left(\frac{8}{3} \pi m y\left(\frac{2 k_{B}}{m}\right)^{3 / 2}\right)^{2}\left[-\frac{15}{2} i_{4}(\alpha) \frac{d i_{2}(\alpha)}{d \alpha}-3\left(\frac{d i_{4}(\alpha)}{d \alpha}\right)^{2}\right] .
$$

Hence, the validity of $(33)_{3}$ is proven if the term in (63) inside the square bracket is positive. First of all, from $(29)$ we have $-5 i_{4}(\alpha) / 2=\frac{d i_{6}(\alpha)}{d \alpha}$, so condition $(33)_{3}$ holds if

$$
I=\left[\frac{d i_{6}(\alpha)}{d \alpha} \frac{d i_{2}(\alpha)}{d \alpha}-\left(\frac{d i_{4}(\alpha)}{d \alpha}\right)^{2}\right]>0
$$

furthermore, $I$ can be rewritten explicitly as

$$
I=\int_{0}^{\infty} \int_{0}^{\infty} \frac{\partial f_{\mathrm{E}}(x, \alpha)}{\partial \alpha} \frac{\partial f_{\mathrm{E}}(x, \alpha)}{\partial \alpha}\left(x^{6} y^{2}-x^{4} y^{4}\right) \mathrm{d} x \mathrm{~d} y .
$$

Since the expression of the integrand in (65) can be rewritten also exchanging the integration variables $x$ and $y$, it holds

$$
\begin{aligned}
& I=\frac{1}{2} \int_{0}^{\infty} \int_{0}^{\infty} \frac{\partial f_{\mathrm{E}}(x, \alpha)}{\partial \alpha} \frac{\partial f_{\mathrm{E}}(x, \alpha)}{\partial \alpha}\left(x^{6} y^{2}-x^{4} y^{4}+y^{6} x^{2}-x^{4} y^{4}\right) \mathrm{d} x \mathrm{~d} y= \\
& =\frac{1}{2} \int_{0}^{\infty} \int_{0}^{\infty} \frac{\partial f_{\mathrm{E}}(x, \alpha)}{\partial \alpha} \frac{\partial f_{\mathrm{E}}(x, \alpha)}{\partial \alpha} x^{2} y^{2}\left(x^{2}-y^{2}\right)^{2} \mathrm{~d} x \mathrm{~d} y .
\end{aligned}
$$

The integrand in (66) is a non-negative function; moreover, its support has clearly a non-zero measure in $R^{2}$, therefore its double integral is strictly positive $(I>0)$. 
When the gas is completely degenerate, that is to say when $\alpha \rightarrow-\infty$ and when $I_{n}(\alpha) \simeq \sqrt{-\alpha}^{n+1}$, the integrand function vanishes and the theorem is no more valid.

We have shown that the entropy density is concave at equilibrium. The same technique as in $(64,65)$ can be used to show that $-\left(h-h_{\mathrm{E}}\right)$ is a positive definite quadratic form. Hence, by continuity arguments, one can conclude that $h$ has to be concave in a neighborhood of the equilibrium state.

\section{Appendix B}

In this appendix we present some results aimed to verify the hyperbolicity property of the model. Following the definition in Section 5, we have preliminarily to show that $A^{0}$ is a non-singular matrix and this is true thanks to the proof of Theorem 1 .

Then, we focus on (51) in order to verify that the eigenvalues are real and there is a set of linearly independent right eigenvectors. Due to the linearization of the system the matrix $\mathbf{A}^{\mathbf{i}}$ does not depend on $J_{i}$ and $q_{i}$. The charachteristic polynomial is a biquadratic form, of the type: $\gamma_{1} x^{4}+\gamma_{2} x^{2}+\gamma_{3}=0$. From Appendix A we already know that $\gamma_{1}=I_{4} I_{0}-\left(I_{2}\right)^{2}>0$ if the gas is not completely degenerate. Permuting the integration variables with the same method as in Appendix A, we show that

$$
\begin{aligned}
& \gamma_{2}=\frac{1}{3}\left(I_{2} I_{4}-I_{0} I_{6}\right)= \\
& =-\frac{2}{3} \int_{0}^{\infty} \int_{0}^{\infty} \frac{\partial f_{\mathrm{E}}(x, \alpha)}{\partial \alpha} \frac{\partial f_{\mathrm{E}}(x, \alpha)}{\partial \alpha} x^{2} y^{2}\left(x^{2}-y^{2}\right)^{2}\left(x^{2}+y^{2}\right) \mathrm{d} x \mathrm{~d} y<0,
\end{aligned}
$$

and

$$
\begin{aligned}
& \gamma_{3}=\frac{1}{9}\left(I_{2} I_{6}-\left(I_{4}\right)^{2}\right)= \\
& =\frac{2}{9} \int_{0}^{\infty} \int_{0}^{\infty} \frac{\partial f_{\mathrm{E}}(x, \alpha)}{\partial \alpha} \frac{\partial f_{\mathrm{E}}(x, \alpha)}{\partial \alpha} x^{4} y^{4}\left(x^{2}-y^{2}\right)^{2} \mathrm{~d} x \mathrm{~d} y>0,
\end{aligned}
$$

if the gas is not completely degenerate.

We conjecture that under the same conditions $\gamma_{2}^{2}-4 \gamma_{1} \gamma_{3}>0$. This idea is supported by several numerical evaluations. If the conjecture is true, we have 4 distinct real eigenvalues (two positive and two negative) and the hyperbolicity requirement is satisfied. In the case of complete degeneration, when $I_{n}(\alpha) \simeq \sqrt{-\alpha}^{n+1}$, all coefficients of (51) vanish identically, so that no sound can propagate in that case [4].

\section{Appendix C}

Here, we list the principal integral used to prescribe the boundary conditions as described in section 7 . For more clarity, we recall that the symbol $\int \cdot \mathrm{d} \mathbf{c}$ 
represents a triple integral, so, here, we consider a transformation in spherical coordinates:

$$
\begin{aligned}
& \int_{c_{1}>0} f_{\mathrm{E}}(T) c^{2 n} c_{1}^{l} \mathrm{~d} \mathbf{c}=\int_{0}^{\infty} \int_{0}^{2 \pi} \int_{0}^{\pi} \frac{y}{e^{\alpha+\frac{c^{2}}{\theta}}+1} c^{2 n} c^{l} \cos ^{l}(\vartheta) c^{2} \sin (\vartheta) \mathrm{d} \vartheta \mathrm{d} \Phi \mathrm{d} c= \\
& =\frac{2 \pi y}{l+1} \theta^{(2 n+l+3) / 2} i_{2 n+l+2}(\alpha) . \\
& \int_{c_{1}>0} \frac{y e^{\alpha+\frac{c^{2}}{\theta}}}{\left(e^{\alpha+\frac{c^{2}}{\theta}}+1\right)^{2}} c^{2 n} c_{1}^{l} \mathrm{~d} \mathbf{c}=\int_{0}^{\infty} \int_{0}^{2 \pi} \int_{0}^{\pi} \frac{y e^{\alpha+\frac{c^{2}}{\theta}}}{\left(e^{\alpha+\frac{c^{2}}{\theta}}+1\right)^{2}} c^{2 n} c^{l} \cos ^{l}(\vartheta) c^{2} \sin (\vartheta) \mathrm{d} \vartheta \mathrm{d} \Phi \mathrm{d} \mathbf{c}= \\
& =\frac{\pi y(2 n+l+1)}{l+1} \theta^{(2 n+l+3) / 2} i_{2 n+l}(\alpha) .
\end{aligned}
$$

\section{Acknowledgment}

This paper was supported by GNFM-INdAM.

\section{References}

1. A. Sommerfeld, Vorlesungen über theoretische Physik 5 Thermodynamik und Statistik, Dietrichshe Verlagbuchhandlung, Wiesbaden (1952).

2. A. Sommerfeld, H. Bethe, Elektronentheorie der Metalle. Handbuch der Physik. XIV, Springer, Heidelberg (1956).

3. I. Müller, On the frame dependence of electric current on heat flux in metals, Acta Mechanica, 24, p. 117-128 (1976).

4. I. Müller, T. Ruggeri, Rational Extended Thermodynamics, Springer, New York, (1998).

5. T. Ruggeri, M. Sugiyama, Rational extended thermodynamics beyond the monatomic gas, Springer, New York (2015).

6. S. Pennisi, T. Ruggeri, Relativistic Extended Thermodynamics of Rarefied Polyatomic Gases, Annals of Physics 377, p.414-445 (2017) .

7. M. Trovato, Quantum Maximum Entropy Principle and Quantum Statistics in Extended Thermodynamics, Acta Applicandae Mathematicae 132, p.605-619 (2014).

8. T. Ruggeri, Extended Thermodynamics with Eight Fields, Bulletin of the Technical University of Instabul. Special issue dedicated to Erdogan Suhubi 47, p. 161-170, (1994).

9. I. Liu, Method of Lagrange multipliers for exploitation of theentropy principle, Arch. Ration.Mech.and Anal., 46, p.131-148, (1972).

10. T. Ruggeri, A. Strumia, Main field and convex covariant density for quasilinear hyperbolic systems. Relativistic fluid dynamics, Ann. Inst. H. Poincar, Sect A 34, p. 65-84 (1981).

11. T. Ruggeri, M.Trovato, Hyperbolicity in extended thermodynamics of Fermi and Bose gases, Contin. Mech. Thermodyn. 16, p. 551-576 (2004).

12. H. Grad, On the kinetic theory of rarefied gases, Comm. Pure Appl. Math. 2, p. 331-407 (1949).

13. E. Barbera, F. Brini, Heat transfer in a binary gas mixture between two parallel plates: An application of linear extended thermodynamics, Acta Mech. 220, p.87-105 (2011).

14. E. Barbera, F. Brini, Heat transfer in gas mixtures: Advantages of an extended thermodynamics approach, Physics Letters A 375, p. 827-831 (2011). 
15. T. Arima, E. Barbera, F. Brini, M.Sugiyama, The role of the dynamic pressure in stationary heat conduction of a rarefied polyatomic gas, Physics Letters A 378, p.26952700 (2014).

16. E. Barbera, F. Brini, Stationary heat transfer in helicoidal flows of a rarefied gas, EPL 120, 34001 (2017).

17. K. Fuchs, The conductivity of thin metallic films according to the electron theory of metals, Math. Proceed. of the Cambridge Phil. Soc. 34, p. 100-108 (1938). 